Proceedings

\title{
Indicators for monitoring the Sustainability Transformations in Territorial Planning
}

1 Affiliation 1; University of L'Aquila, Department of Civil, Construction-Architectural and Environmental Engineering; alessandro.marucci@univaq.it; lorena.fiorini@univaq.it; lucia.saganeiti@univaq.it; rosamarina.donolo@univaq.it

* Correspondence: University of L'Aquila, Department of Civil, Construction-Architectural and Environmental Engineering; alessandro.marucci@univaq.it)

\begin{abstract}
This research aims to identify a set of indicators for the Abruzzo Region to monitor the sustainability of the transformations in territorial planning, in order to focus the "position" of the Region with respect to the goals of the National Sustainable Development Strategy (NSDS) and the 17 Goals of the 2030 Agenda. The proposed methodology consists of: a coherence analysis with the aim of verifying the existence of a convergence between the goals and the general strategies of the Abruzzo Region's Plans, and the Goals/Principles of environmental sustainability derived from the NSDS; a recognition of the sustainability indicators already developed by various public/private institutions, at national level, in order to select the most suitable sustainable development indicators for the Abruzzo Region.
\end{abstract}

Keywords: Sustainable Development Goals; Indicators; Urban transformations; Abruzzo Region; Sustainability.

\section{Introduction}

The land transformation phenomena are acquiring in recent years increasingly peculiar connotations characterized by a growing speed of change that requires a continuous monitoring [1-3]. The need to measure these transformations emerges, together with the need to control them, by limiting or, where necessary, by inverting the processes. This research aims to address a very topical issue, the 2030 Agenda for Sustainable Development [4], focusing on the sustainability indicators and how they represent an indispensable tool for monitoring transformations and the impact of human activities interacting with Natural Capital $[5,6]$. This contribution proposes an analysis of the regional planning of the Abruzzo Region in order to determine its positioning with respect to the objectives of the National Sustainable Development Strategy (NSDS); moreover, this contribution proposes to identify a set of indicators to evaluate the urban transformations performance. For this purpose, the set of indicators represents a first dynamic and updatable outline of the needs of regional planning with respect to the adaptation to the NSDS. At a national level, the sustainability indicators proposed by the Italian Statistical Institute (ISTAT) [7] on the basis of the NSDS and those proposed by the Institute for Environmental Protection and Research (ISPRA) [8], are a good starting point for assessing the sustainability of transformations. The main results of this work, centered on the regional planning of the Abruzzo Region, show a continuous link to goals 11, 13 and 15 that are related to life on earth, climate actions and the pursuit of performance to build sustainable cities and communities. This highlights the need to address new challenges related to the environment through an integrated approach between ecosystem functions and services, sustainability principles and urban resilience. 
Following this approach, in the current work, a coherence analysis has been carried out through the developing of coherence matrices of the Plans acting on the regional territory with respect to the Sustainable Development Goals (SDGs). The coherence matrices are configured as a regional governance tool as they allow to take a picture of the current state of regional planning, positioning it with respect to NSDS and as they allow to update and improve its performance to achieve the goals set by AGENDA 2030.

\section{Regional Plans and NSDS: A Coherence Analysis}

In order to determine the positioning of regional planning with respect to the SDGs, a coherence analysis has been conducted. The purpose was to verify the existence of convergence, explicit or implicit, between the objectives of 16 regional Plans and the 17 Goals of the AGENDA 2030. Specifically, the analysis of external coherence was useful to assess the compatibility of the objectives and general strategies of the Plans with the objectives/principles of environmental sustainability, taken from the NSDS. Three types of coherence were recognized: (i) direct coherence between the strategies of the Plan and the NSDS i.e., explicit reference is made in the plan to the NSDS or AGENDA 2030, indirect coherence between Plan strategies and the NSDS i.e., in the Plan there is no explicit reference to the NSDS but its goals are implicitly referred to in the Plan strategies; and uncertain coherence because it depends on the way in which measures are implemented in the different urban planning tools. In addition, the case of incomparability has also been identified.

The results obtained are summarized in a matrix where: in columns are reported the 5 strategic areas of NSDS (People, Planet, Prosperity, Peace, Partnership) and the system of sustainability vectors; in rows the 16 regional Plans considered. The sustainability vectors are defined in the NSDS as a transversal action to the strategic areas and as fundamental components to activate, manage, monitor and guide an integrated approach between ecosystem services, urban transformation and sustainability towards the construction of policies, Plans and specific national projects. The regional Plans currently in force have been approved after the 2000s (except for the Regional Landscape Plan approved in the 1990s) however, they were drafted on the basis of obsolete regional and national legislation. For example, the Regional Reference Framework (QRR) was approved in 2000 but was drafted according to a 1983 regional law or the Gran Sasso e Monti della Laga National Park Plan was approved in 2020 and was drafted according to a 1991 law. It is evident, that all the regional planning is lacking clear and explicit references to current environmental and sustainability policies and, therefore, in the coherence matrix, for the current state of planning, will never result in a status of direct coherence.

Figure 1 shows an extract of the coherence matrix where, in correspondence of each level of coherence, the Goals related to the choices of the NSDS have been inserted. It is evident that for the Planet and the Partnership areas, there is a clear convergence since the SDGs are the natural evolution of the univocal definition of universally recognized principles such as halting the loss of biodiversity, promoting a fair and rational management of natural resources, combating poverty and social inequality.

Therefore, considering the initial results, it is possible to promote a first level of implementation of the Regional Strategy by updating the current framework according to the definitions dictated by the UN. This reinforces the role of the 2030 Agenda as a condenser of current sustainability policies. 


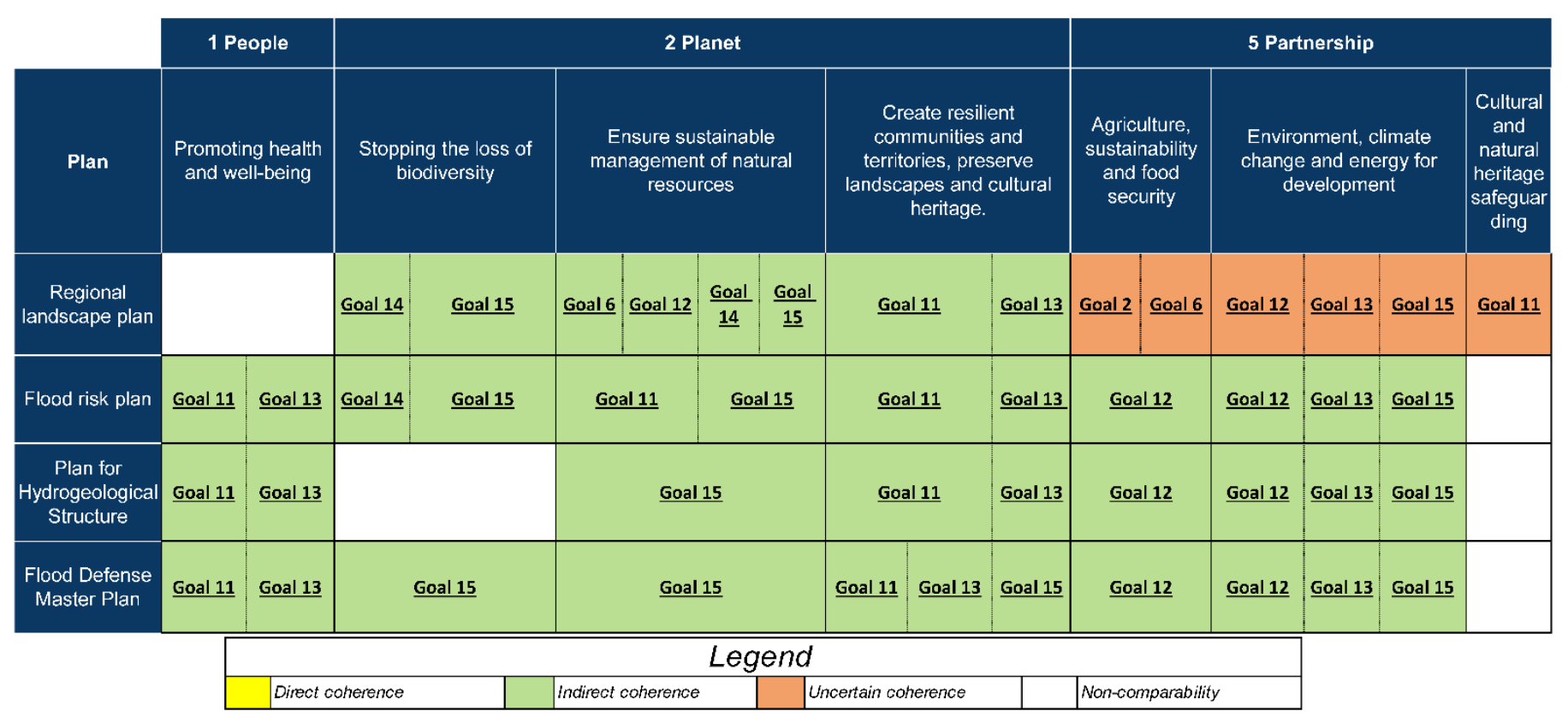

Figure 1. Coherence matrix with the goals indivduated for each level of coherence and for each plan.

\section{Indicators for monitoring the Sustainability of Transformations}

In Italy, the urban and territorial planning is regulated by the national law n. 1150 of 1942 and by numerous regional laws developed after 1970. However, it should be noted that effective procedures for measuring, monitoring and making analytical diagnosis of the effects of operational tools for territorial governance have rarely been used. Currently, the use of indicators in the field of environmental and social policies is becoming more and more frequent. In fact, in recent years, there has been a clear change in the approach used by the various components involved in planning. Also due to the growing need to direct the analyses towards the parametric and formalized restitution of phenomena in order to increase the ability to interpret and evaluate the results obtained. Moreover, an efficient methodology based on the use of indicators should be developed through the following stages: (i) Identification of the parameter set; (ii) Detection and measurement of each indicator; (iii) Diagnostic interpretation of the phenomena; (iv) Identification of the cutoff values, which are fundamental for carrying out the control of the phenomena; (v) Monitoring of the effects. In this first phase, the work developed for the Abruzzo Region concerned only the phase of identifying the set of indicators, with respect to the five stages previously listed. A fundamental step of the work was to start a survey of the sustainability indicators already developed and in use by various public and/or private institutions. Subsequently, in order to identify a set of suitable/usable indicators, it has been decided to consider only the set of indicators provided by the institutional sources ISTAT and ISPRA, with reference to the following documents:

- SDGs 2020 Report - Statistical information for the 2030 Agenda in Italy (ISTAT, 2020 - link: https://www.istat.it/it/archivio/242819);

- Annual Report of environmental data 2020 (ISPRA, 2020 - link: https://bit.ly/320gdyN).

The indicators coming from the two main data sources (ISTAT and ISPRA) have been homogenized. In particular, the ISPRA indicators were related to the 5 strategic areas and vectors of sustainability identified by the NSDS.

According to the specific Goal and the environmental theme (biosphere, economy, environment, waste, energy, etc.), the most relevant strategic area was associated to each indicator. After this homogenization, it was possible to select the most useful indicators by setting up appropriate filters based on the Goals and strategic areas. In this way, the matrix of indicators was compiled. From the previous matrix of coherence and objectives, 
for each level of coherence have been identified: (i) the Goals to which the specific choice of the strategic area of the NSDS refers; (ii), the most useful indicators for monitoring purposes have been selected from the built set of indicators, based on: the Goals, the strategic area and the Plan considered. In the matrix, many indicators are repeated since they are useful for the monitoring of different Plans, therefore, the total number of unique indicators identified in this first phase is 66. Among these indicators, it would be possible to make a further selection by considering, for example, the indicators that allow to measure the performance of several Plans simultaneously. This concept is well summarized in the diagram created by the authors available at the following link: https://bit.ly/3ywhDvx.

\section{Conclusions}

In this research, the use of ISTAT and ISPRA indicators datasets comes from the need to determine already in the early stages of the development of the Regional Strategy a common and institutional language in order to maximize the interoperability and the possibility of comparison with other territorial and administrative contexts. The main results achieved, show, a continuous reference to the Goals 11, 13, and 15 referring to sustainable cities and communities, climate actions and life on earth, that is evident, in the coherence matrices of the 16 Plans. Precisely these three goals contain the new demands for a trend reversal with respect to the dynamics of transformation and use of land and the integrated protection of biodiversity. Therefore, on one hand the above-mentioned universal principles are present in the territorial government tool scenario, on the other hand there is still no clarity and uniformity in the formulation of policy makers intentions.

In conclusion, the proposed set of indicators represents a first dynamic and updatable outline of the needs of regional planning with respect to adaptation to NSDS, but still needs to undergo to a review process and to a continuous comparison with the competent authorities, in order to adapt to their specific needs.

Funding: This research was funded by Agreement for the granting of a research grant aimed at revising the system of regional indicators as part of the implementation of regional strategies for Sustainable Development. (Article 15 of Law 241/1990 and subsequent amendments), funded by the Abruzzo Region (Italy).

Conflicts of Interest: The authors declare no conflict of interest.

\section{References}

1. EEA Land accounts for Europe 1990-2000: Towards integrated land and ecosystem accounting; 2006; Vol. 11; ISBN 9291678880.

2. Decoville, A.; Schneider, M. Can the 2050 zero land take objective of the EU be reliably monitored? A comparative study. J. Land Use Sci. 2015, 11, 1-19, doi:10.1080/1747423X.2014.994567.

3. European Commission No net land take by 2050?; 2016;

4. Sachs, J.; Kroll, C.; Lafortune, G.; Fuller, G.; Woelm, F. Sustainable Development Report 2021. Sustain. Dev. Rep. 2021 2021, doi:10.1017/9781009106559.

5. Shen, L.Y.; Jorge Ochoa, J.; Shah, M.N.; Zhang, X. The application of urban sustainability indicators - A comparison between various practices. Habitat Int. 2011, 35, 17-29, doi:10.1016/j.habitatint.2010.03.006.

6. Science for Environment Policy Indicators for Sustainable Cities ; 2017;

7. ISTAT Rapporto SDGs 2020. Informazioni statistiche per l'Agenda 2030 in Italia; 2020;

8. ISPRA Il Catalogo obiettivi - indicatori 2011 - Available online: https://www.isprambiente.gov.it/it/attivita/autorizzazioni-evalutazioni-ambientali/valutazione-ambientale-strategica-vas/il-catalogo-obiettivi-indicatori-2011 (accessed on Jul 12, 2021). 